\title{
Primary Synovial Sarcoma of the Kidney: A Case Report
}

\author{
Nouha Ammar'1, Imene Chabchoub ${ }^{1}$, Rihab Ben Jaafar'1, Hiba Sboui1 ${ }^{*}$, Makram Hochlef1, \\ Faten Ezzaairi', Sami Sfar'2, Imtinane Belaid1, Leila Ben Fatma1, Manel Njim³, Nadia Bouzid4, \\ Mohsen Belguith' ${ }^{2}$, Rzig Ahmed5, Zakhama Abdelfateh ${ }^{3}$, Slim Ben Ahmed1
}

${ }^{1}$ Medical Onocology Department, University Hospital Farhat Hached, Sousse, Tunisia

${ }^{2}$ Pediatric Surgery Department, University Hospital Fatouma Bourguiba, Monastir, Tunisia

${ }^{3}$ Anathomopathology Department, University Hospital Fatouma Bourguiba, Monastir, Tunisia

${ }^{4}$ Radiotherapy Department, University Hospital Farhat Hached, Sousse, Tunisia

${ }^{5}$ Radiology Department, University Hospital Fatouma Bourguiba, Monastir, Tunisia

Email: *sboui-hiba2006@hotmail.com

How to cite this paper: Ammar, N., Chabchoub, I., Jaafar, R.B., Sboui, H., Hochlef, M., Ezzaairi, F., Sfar, S., Belaid, I., Fatma, L.B., Njim, M., Bouzid, N., Belguith, M., Ahmed, R., Abdelfateh, Z. and Ahmed, S.B. (2021) Primary Synovial Sarcoma of the Kidney: A Case Report. Journal of Cancer Therapy, 12, 579-584. https://doi.org/10.4236/jct.2021.1210049

Received: September 16, 2021

Accepted: October 26, 2021

Published: October 29, 2021

Copyright $\odot 2021$ by author(s) and Scientific Research Publishing Inc. This work is licensed under the Creative Commons Attribution International License (CC BY 4.0).

http://creativecommons.org/licenses/by/4.0/

(c) (i) Open Access

\begin{abstract}
Synovial sarcoma originating from the kidney is extremely rare. A 13-year-old girl presented with a mild left flank pain of one-week duration, with no associated history of hematuria or any other systemic symptoms. Computed tomography $(\mathrm{CT})$ demonstrated a $6 \times 13 \times 9 \mathrm{~cm}$ mass in the left kidney. No soft tissue or extrarenal masses were identified. The patient received a combined of treatment with doxorubicin and ifosfamide. A radical nephrectomy was performed in the left kidney with no complications. Postoperative pathology revealed post-chemotherapy residue of monophasic spindle cell synovialosarcoma of the left kidney. She received a combined treatment with doxorubicin and ifosfamide in concomittance with external radiation therapy. The patient was re-examined 4 months after surgery. An abdominal and pulmonary CT found no recurrence or metastasis.
\end{abstract}

\section{Keywords}

Synovial Sarcoma, Kidney

\section{Introduction}

Synovial Sarcomas (SS) are a group of Soft Tissue Sarcomas (STS) affecting mainly young adults. The most common site of occurrence is in extremities [1].

Primary Synovial Sarcoma (PSS) of the kidney is a recently described entity [2]. 
Fewer than 50 cases of primary renal synovial sarcoma are reported to date [3].

This tumor presents a diagnostic dilemma because it is quite difficult to differentiate it from other renal neoplasms, such as metastatic sarcoma, renal cell carcinoma with sarcomatoid differentiation, which may have similar histological features.

In the present report, we present the case of a 13-year-old girl who was diagnosed with primary renal synovial sarcoma.

\section{Case Report}

A 13-year-old female was presented with a mild left flank pain of one-week duration, with no associated history of hematuria or any other systemic symptoms.

Renal ultrasound showed a large left upper polar renal mass $16 \times 13 \times 9 \mathrm{~cm}$, hypoechoic, homogeneous, well limits, presenting rich vascularization on color Doppler.

The diagnosis of nephroblastoma has been suggested.

Computed tomograpgy of the abdomen and pelvis showed a left retroperitoneal mass probably renal origin measuring $14 \times 10 \times 11.5 \mathrm{~cm}$ associated with retroperitoneal adenomegaly.

A CT scan of the chest did not reveal metastatic disease (Figure 1).

The patient underwent a kidnet biopsy which revealed a histological and immunohistochemical monophasic spindle cell synovialosarcoma of left kidney.

On immunohistochemistry, the tumor cells showed an intense and diffuse nuclear activity for TLE1, focal positivity for cytokeratin AE1/AE3.

WT1 was focally positive but this positivity is not specific (Figure 2).

According to these findings, a diagnosis of primary monophasic SS of the kidney was made.

A complete tumor resection with node dissection cannot be obtained, the patient was referred to our department of pediatric oncology in sousse for the neoadjuvant chemotherapy.

She received chemotherapy with Doxorubicin $37.5 \mathrm{mg} / \mathrm{m}^{2} /$ day for 2 days and Ifosfamide $3 \mathrm{~g} / \mathrm{m}^{2} /$ day for 3 days every 21 days for a total of 3 cycles according to EpSSG NRSTS 2005 protocol with good tolerance.

2 weeks after the chemotherapy, the patient had a CT of the abdomen and pelvis which showed a decrease in size of the heterogeneous left renal mass of 8 $\mathrm{cm}$ (vs. $14 \mathrm{~cm}$ ) (estimated at 42\%), clear regression of the effect of mass on the anterior structures previously described with, however, persistence of intimate contact with the tail of the pancreas and the splenic vein.

No retro-peritoneal adenomegaly of significant size.

No new lesion (Figure 3).

The patient was transferred to pediatric surgery department, she underwent exploratory laparotomy that involved the resection of a $10 \mathrm{~cm}$ mass, with a left uretro-nephrectomy (Figure 4). 


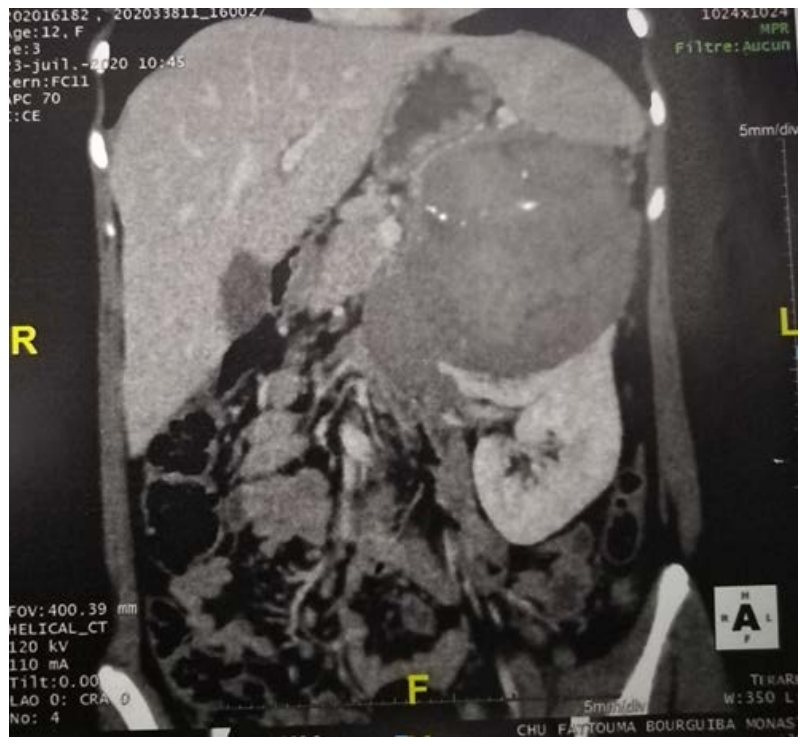

Figure 1. Coronal section of a computed-tomographic scan of the abdomen and pelvis, showing a left retroperitoneal mass with fine calcifications and few hypodense areas of necrosis.

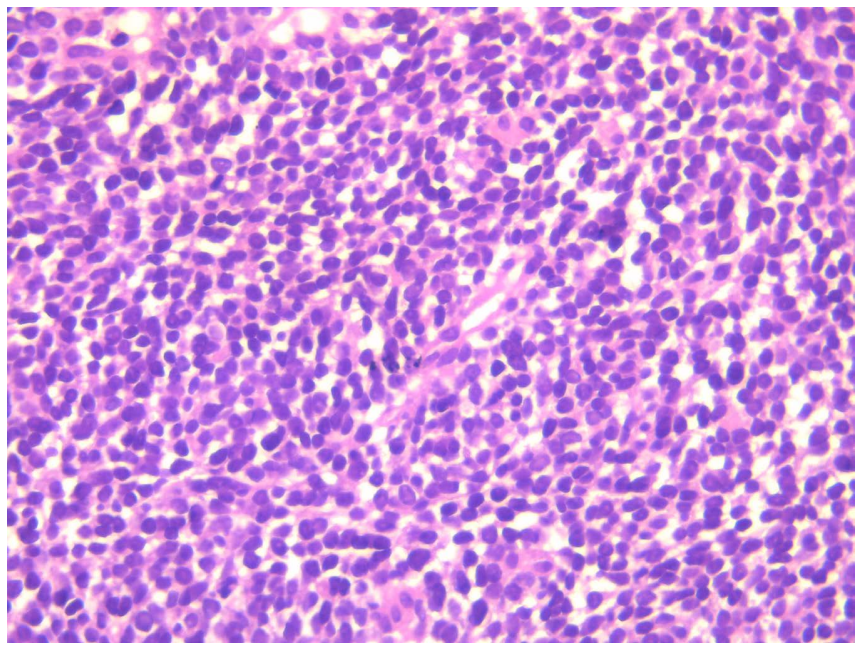

Figure 2. Tumor composed of spindle cells arranged in intersecting fascicles alternating with hypocellular areas.

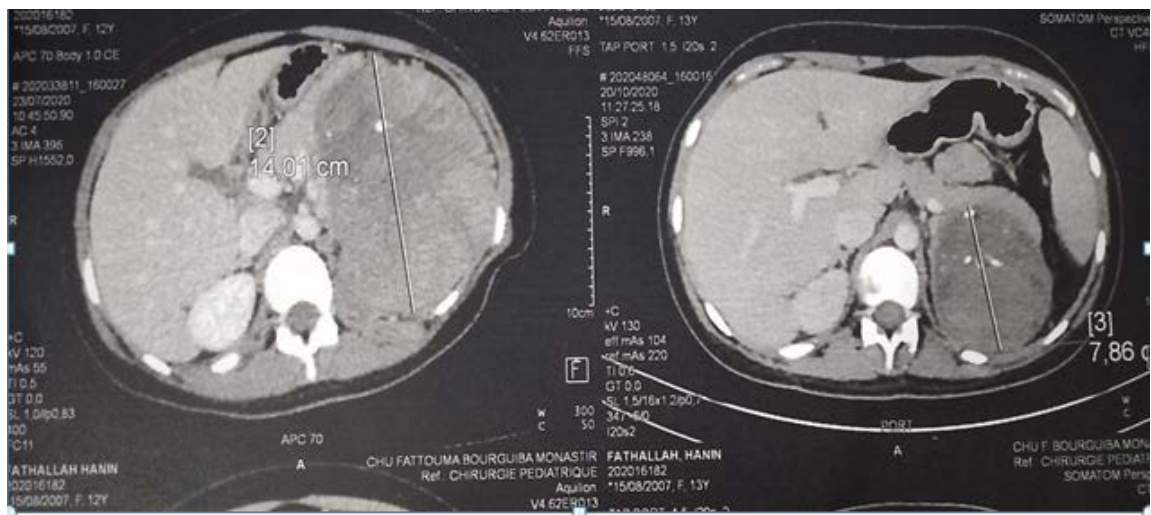

Figure 3. CT image showing marked decrease in tumor size. 


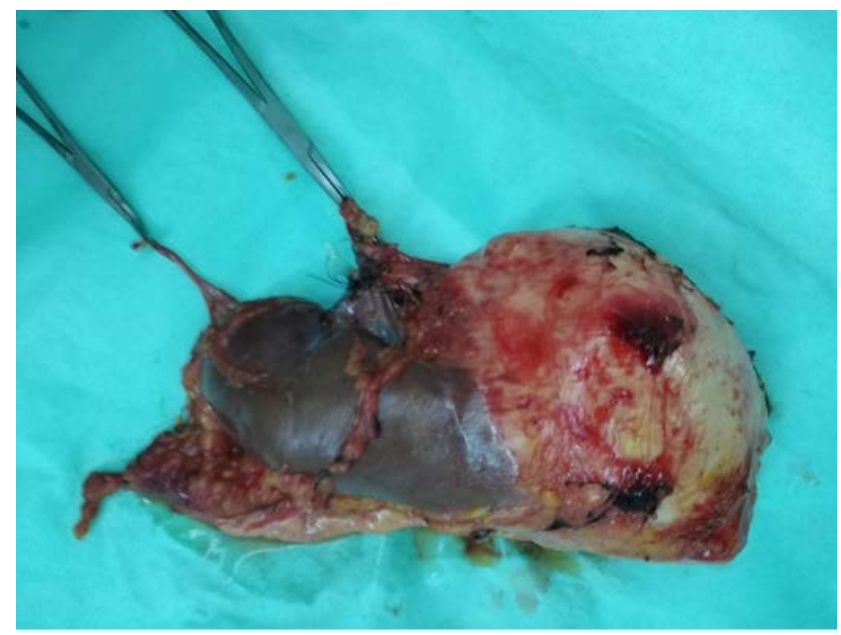

Figure 4. Macroscopically, the tumor mesarures $10 \mathrm{~cm}$ and shows fibro-necrotic changes secondary to chemotherapy.

Histology revealed post-chemotherapy residue of monophasic spindle cell synovialosarcoma of the left kidney.

The tumor measures $10 \mathrm{~cm}$ long and shows fibro-necrotic changes secondary to chemotherapy estimated at $80 \%$ of the tumor volume.

Absence vascular embolus of the hilum vessels.

After recovering from surgery, the patient received one cycle of chemotherapy (Doxorubicin $37.5 \mathrm{mg} / \mathrm{m}^{2} /$ day for 2 days and Ifosfamide $3 \mathrm{~g} / \mathrm{m}^{2} /$ day for 3 days) and 2 cycles of chemotherapy (Ifosfamide alone at $3 \mathrm{~g} / \mathrm{m}^{2} /$ day for 2 days) concomitantly to radiotherapy. She received $45 \mathrm{~Gy}(1.8 \mathrm{~Gy} / \mathrm{d})$ at the 9 th week, concomitantly to 4 th and 5 th cycles with a good tolerance.

After 6 months the patient came for follow up and she was doing well.

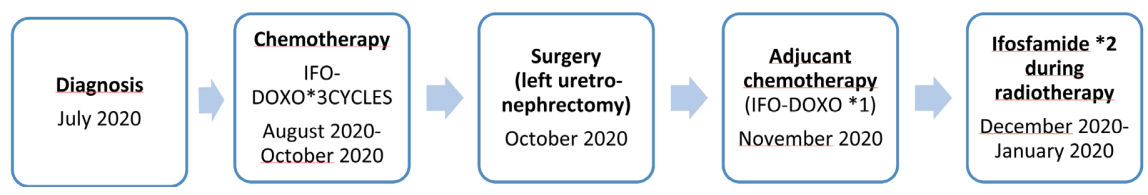

\section{Discussion}

Monophasic synovial sarcoma of the kidney is an extremely rare tumor and less than 50 cases have been described in the literature.

It was first reported in 1999 by Faria et al. [4].

Synovial sarcoma usually involves adolescents and young adults; however, the age at presentation ranges from 17 to 61 years. Diagnosis is difficult due to the rarity of the tumor and its similar presentations as compared to other renal tumors. Differential diagnosis includes Adult Wilms tumor, transitional cell carcinoma, renal cell carcinoma and hemangiopericytoma, congenital mesoblastic nephroma, and primitive neuroectodermal tumor [5].

The patients commonly present with flank pain and/or hematuria. No clinical feature or imaging modality is diagnostic. The CT scan usually reveals a hetero- 
geneously enhancing renal mass and the confirmation of diagnosis is by molecular and cytogenetic analysis. Rarely is the presentation at an advanced stage with caval thrombus and/or metastasis. To the best of our knowledge, approximately three cases of SS with the caval thrombus have been reported previously. In 2007, Tornkvist et al. [6] reported six cases of metastatic disease.

Primary synovial sarcoma occurs in two forms: Biphasic and Monophasic. The primary biphasic synovial sarcoma contains both glandular elements and spindle epithelial cells. The primary monophasic synovial sarcoma is composed of only spindle cells [7].

There are no established guidelines regarding management of this tumor given the limited number of cases reported. Primary surgical treatment is considered to be the treatment of choice; prognosis is poor with this treatment alone. Surgical resection is the mainstay, although surgery alone has a poor prognosis. Reports of sensitivity of this tumor to ifosfamide- and doxorubicin-based chemotherapy do exist but no clear guidelines are available regarding adequate treatment of this rare entity [8].

Radiotherapy is also effective in presence of local spread as it was in our patient [9].

\section{Conclusions}

In conclusion, primary SS of the kidney is an extremely rare disease and preoperative diagnosis is difficult in the absence of specific clinical or imaging findings.

Its diagnosis is based on morphological and molecular studies.

The combination of surgery and chemotherapy has shown positive results. Particularly, the use of Ifosfamide and Doxorubicin is as standard chemotherapy to induce complete remission.

Radiotherapy can be useful as adjuvant therapy in presence of local spread.

\section{Conflicts of Interest}

The authors declare no conflicts of interest regarding the publication of this paper.

\section{References}

[1] Kim, D.-H., Sohn, J.H., Lee, M.C., et al. (2000) Primary Synovial Sarcoma of the Kidney. The American Journal of Surgical Pathology, 24, 1097-1104. https://doi.org/10.1097/00000478-200008000-00007

[2] Gabilondo, F., Rodríguez, F., Mohar, A., Nuovo, G.J. and Domínguez-Malagón, H. (2008) Primary Synovial Sarcoma of the Kidney: Corroboration with In Situ Polymerase Chain Reaction. Annals of Diagnostic Pathology, 12, 134-137. https://doi.org/10.1016/j.anndiagpath.2006.08.005

[3] Ozkan, E.E., Mertsoylu, H. and Ozardali, H.I. (2011) A Case of Renal Synovial Sarcoma Treated with Adjuvant Ifosfamide and Doxorubicin. Internal Medicine, 50, 1575-1580. https://doi.org/10.2169/internalmedicine.50.5224 
[4] Faria, P., Argani, P. and Epstein, J. (1999) Primary Synovial Sarcoma of the Kidney: A Molecular Subset of So Called Embryonal Renal Sarcoma. The American Journal of Surgical Pathology, 12, $94 \mathrm{p}$.

[5] Shao, L., Hill, D.A. and Perlman, E.J. (2004) Expression of WT-1, Bcl2 and CD34 by Primary Renal Spindle Cell Tumors in Children. Pediatric and Developmental Pathology, 7, 577-582. https://doi.org/10.1007/s10024-004-4037-6

[6] Tornkvist, M., Wedje, J., Ahlan, J., Brodin, B. and Larsson, O. (2004) A Novel Case of Synovial Sarcoma of Kidney: Impact of SS18/SSX Analysis of Renal Hemangiopericytoma Like Tumors. Diagnostic Molecular Pathology, 13, 47-51. https://doi.org/10.1097/00019606-200403000-00008

[7] Skytting, B., Nilsson, G., Brodin, B., Xie, Y., Lundeberg, J., Uhlen, M., et al. (1999) A Novel Fusion Gene, SYT-SSX4, In Synovial Sarcoma. Journal of the National Cancer Institute, 91, 974-975. https://doi.org/10.1093/jnci/91.11.974

[8] Park, S.-J., Kim, H.-K., Kim, C.-K., Park, S.-K., Go, E.-S., Kim, M.-E. and Hong, D.S. (2004) A Case of Renal Synovial sarcoma: Complete remission was induced by chemotherapy with Doxorubicin and Ifosamide. The Korean Journal of Internal Medicine, 19, 62-65. https://doi.org/10.3904/kjim.2004.19.1.62

[9] Kawai, A., Woodruff, J., Healey, J.H., et al. (1998) SVT-SSX Gene Fusion as a Determinant of Morphology and Prognosis in Synovial Sarcoma. The New England Journal of Medicine, 338, 153-160. https://doi.org/10.1056/NEJM199801153380303 